\title{
Risk factors of gestational diabetes mellitus: A hospital-based pair- matched case-control study in coastal South India
}

\author{
B Unnikrishnan, ${ }^{1,2}$ MD; P Rathi, ${ }^{1}$ MD; S K Bhat, ${ }^{1}$ MBBS; P H Nayak, ${ }^{1}$ MBBS; N Ravishankar, ${ }^{1}$ MBBS; A Singh, ${ }^{1}$ MBBS; \\ O Praveen, ${ }^{1} \mathrm{MBBS}$
}

${ }^{1}$ Kasturba Medical College, Mangalore, Manipal Academy of Higher Education, Manipal, India

${ }^{2}$ Datta Meghe Institute of Medical Science, Wardha, India

Corresponding author: P Rathi (priya.rathi@manipal.edu)

\begin{abstract}
Background. In the light of the rise in prevalence of gestational diabetes mellitus (GDM) and the importance of early suspicion and prompt diagnosis, different regions have reported different risk factors. This warrants multiple regional studies in sub-populations and diverse geographic areas to quantify determinants of GDM.

Objective. To determine risk factors of GDM.

Methods. A case-control study was conducted in a tertiary care maternity hospital in coastal South India based on pregnancy records of all those women diagnosed with GDM who received in-patient care between January 2018 and December 2018. There were 131 cases pairmatched for age in the ratio of 1:1 with 131 controls.

Results. Women from rural areas and those with a monthly income $\leq$ INR20 000, body mass index (BMI) $>23 \mathrm{~kg} / \mathrm{m}^{2}$, polyhydramnios (a trend), pregnancy-induced hypertension (a trend), oligohydramnios (a trend) and a gap between pregnancies of $<2$ years had a higher risk. As regards previous pregnancies, women with poor obstetric outcomes - miscarriage and pre-term labour - had a trend to greater risk in comparison with those who had either had vaginal delivery or caesarean section.

Conclusion. BMI $>23 \mathrm{~kg} / \mathrm{m}^{2}$ and a spacing gap of $<2$ years between pregnancies were significant risk factors, whereas those having had a vaginal birth in previous pregnancies in this population had a diminished risk of developing GDM. A larger study is required to demonstrate significance in other factors.
\end{abstract}

S Afr J Obstet Gynaecol 2020;26(1):13-17. https://doi.org/10.7196/SAJOG.2020.v26i1.1518

Gestational diabetes mellitus (GDM) is defined as 'any degree of glucose intolerance with onset or first recognition during pregnancy. ${ }^{[1]}$ This definition is valid both when diet modification is sufficient and when insulin or other agents are used for treatment, whether or not the condition persists after pregnancy. ${ }^{[1,2]}$ Hyperglycaemia has many effects on pregnancy and $\sim 16.9 \%$ of all pregnancies are estimated to be affected by it. ${ }^{[3]}$ The distribution in the world of GDM is highest in the Asia-Pacific region. This may be due to a tendency of the Asian population towards abdominal obesity, low muscle mass and increased insulin resistance in contrast to their western counterparts. ${ }^{[4]}$

A meta-analysis performed on an Asian population reported a mean prevalence of GDM of $11.5 \%{ }^{[5]}$ The highest prevalence is reported in Southeast Asia, where up to 1 in 7 births may be affected by GDM. The Indian population has a higher prevalence rate of GDM in comparison with some other populations of Southeast Asia. ${ }^{[3]}$ The number of Indians with diabetes increased from 26 million in 1990 to 65 million in $2016,{ }^{[6]}$ and the estimated prevalence for 2025 is 79.4 million. ${ }^{[7]}$ The prevalence of gestational diabetes across India has been reported to range from $3.8 \%$ in Kashmir $^{[8]}$ to $41 \%$ in Lucknow. ${ }^{[9]}$ As a public health concern, GDM is increasing globally, as well as in India.

Risk factors that lead to GDM can be divided into determinants antecedent to the current pregnancy and those that occur during the pregnancy. Familial history of GDM, irregular menstrual history, maternal age, prevalence of obesity, history of fetal anomalies, stillbirth, polycystic ovarian syndrome, infertility, recurrent urinary tract infections, multiparity, high body mass index (BMI), and certain ethnicities, including Asian, fall under the first category of risk factors. Excessive pregnancy weight gain, macrosomia, pre-eclampsia and lack of physical activity fall under risk factors that occur during the course of the pregnancy. ${ }^{[10,11]}$

GDM has long-term and short-term health-related effects for both the affected women and their offspring. In women, it is associated with a greater risk of gestational hypertension, pre-eclampsia and complications during delivery and the postnatal period. In the fetus it may lead to either macrosomia or small-for-gestational-age infants, and predispose to birth trauma, hypoglycaemia and other metabolic disturbances. The possibility of consequences after birth is still under

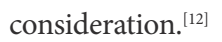

The clinical recognition of GDM is important as it helps to alleviate associated perinatal morbidity and mortality. It necessitates diet or drug therapy, such as insulin and antepartum fetal and maternal surveillance. ${ }^{[2]}$

International health organisations have emphasised the need for developing national plans to improve the quality of healthcare. Nonetheless, constant surveillance of healthcare quality poses a significant challenge in many countries. In this context, data regarding risk factors of GDM are necessary to carry out rational planning and distribution of resources in order to develop efficient preventive strategies. In India, different regions have reported broadly different risk factors. 
Based on our literature review, we found that in India, a study performed in Kerala by Bhat et al. ${ }^{[13]}$ reported an irregular menstrual cycle, treatment for infertility, a family history of diabetes, history of diabetes in mother, previous pregnancy losses and previous GDM as significant risk factors for GDM. A study by Arora et al. ${ }^{[12]}$ in Punjab reported urban habitat, illiteracy, non-vegetarianism, increased BMI and short adult height as independent risk factors of GDM. Gopalakrishnan et al. ${ }^{[14]}$ in Delhi reported pre-pregnancy BMI $>23 \mathrm{~kg} / \mathrm{m}^{2}$, oral contraceptive pill (OCP) use for more than 5 years, physical activity (sedentary v. moderate (odds ratio (OR) 1.40)) and consumption of food items with high glycaemic index (GI) as risk factors for GDM. In a study in West Bengal, Pramanick and Panda ${ }^{[15]}$ reported GDM mothers were significantly older, and had higher BMI; they also noted significantly higher incidence of lower section caesarean section (LSCS) and an association with hypothyroidism. This difference in risk factors in subtypes of populations and diverse geographic conditions warrants multiple regional studies to quantify determinants of gestational diabetes.

In the light of the rise in prevalence of GDM and the importance of the early detection and prompt diagnosis of GDM, a case-control study was performed to study the risk factors of GDM in women receiving pregnancy care in a tertiary care hospital in Mangalore.

\section{Methods \\ Study design, setting and duration}

This case-control study was conducted at a government tertiary care maternal and child health hospital in Mangalore, coastal South India, from February 2019 to May 2019. The pregnancy records of women who availed themselves of in-patient pregnancy care between January 2018 and December 2018 were included.

Government Lady Goschen Hospital (LGH) is a 260-bedded district-level specialised hospital in Mangalore, district of Dakshina Kannada, Karnataka, which offers service in obstetrics and gynaecology and neonatology. This hospital is a tertiary healthcare facility with its maternity service being a referral centre for high-risk pregnancy care for women throughout Dakshina Kannada and its neighbouring districts.

\section{Ethics approval}

The study protocol was approved by the Institutional Ethics Committee of Kasturba Medical College, Mangalore.

\section{Selection}

Case group: We identified all patients diagnosed as having GDM by manually sorting case sheets from the medical records department of LGH. Women who were pregnant twice during the study period were included only once, using details from their first GDM-affected pregnancy. Women who had either overt diabetes mellitus or any chronic illness were excluded from the study. A total of 131 women diagnosed with GDM met the criteria.

Control group: Information regarding age was collected for women without GDM from hospital medical records. Age-matched records of women who had normal pregnancies were selected, with one control for each study subject. Women with either GDM or impaired glucose tolerance were excluded from the control group. After collecting the data of all cases in 2018, their minimum and maximum ages were recorded; controls were then selected based on the age range of cases.

\section{Data collection tool}

After extensive review of the literature and discussion with experts, a pro forma document containing general information on demographic characteristics, socioeconomic status (according to the Kuppuswamy classification), education level, parity and past history of GDM-affected pregnancy was prepared. Data were collected by investigators according to the pro forma. Table 1 illustrates the variables that were investigated.

\section{Potential confounder}

Age is a potential confounder, based on the literature review; women older than 35 years are known to have an increased risk of GDM. ${ }^{[11]}$ Controls were age-matched.

\section{Diagnostic criteria}

According to the American Diabetes Association (ADA)'s Carpenter-Coustan criteria for diagnosing GDM, all pregnant women were administered a $50 \mathrm{~g}$ oral glucose challenge test (GCT) during the period 24 to 28 weeks of pregnancy as per ADA recommendations. Those with a positive test result (defined as a plasma glucose level $\geq 130 \mathrm{mg} / \mathrm{dL} 1$ hour after the glucose load) were subjected to a 3 -hour $100 \mathrm{~g}$ oral glucose tolerance test (OGTT). The diagnosis of GDM was made if at least two of the following four plasma glucose levels (Carpenter-Coustan criteria: measured fasting $95 \mathrm{mg} / \mathrm{dL}$ and 1-hour $180 \mathrm{mg} / \mathrm{dL}, 2$-hour $155 \mathrm{mg} / \mathrm{dL}, 3$-hour $140 \mathrm{mg} / \mathrm{dL}$ during OGTT) are met or exceeded. ${ }^{[2]}$

\section{Study size}

There were 131 cases with GDM during the study period and they were pair matched with controls in the ratio of 1:1.

\section{Statistical methods}

Data were recorded and analysed using the Statistical Package for Social Sciences (SPSS) version 25.0 (IBM Corp., USA). Descriptive statistics like proportions, mean (standard deviation (SD)), and

\begin{tabular}{ll} 
Table 1. Variables affecting GDM that were investigated \\
\hline Independent variables & \\
\hline Variables & Parameter \\
\hline Sociodemographic variables & \\
Age & rural/urban \\
Residential location & $\leq \mathrm{INR} 20000 />\mathrm{INR} 20000$ \\
Family income & per month \\
& \\
Risk factors based on current pregnancy & \\
BMI at GDM diagnosis & $\leq 23 />23 \mathrm{~kg} / \mathrm{m}^{2}$ \\
GDM diagnosis & on or before 28 weeks/after \\
& 28 weeks of pregnancy \\
Pregnancy-induced hypertension & Yes/No \\
Polyhydramnios & Yes/No \\
Oligohydramnios & Yes/No \\
Risk factors based on previous pregnancies & \\
Mean time between pregnancies & $<2 / \geq 2$ years \\
Abortions & Yes/No \\
Pre-eclampsia & Yes/No \\
Pre-term labour & Yes/No \\
Vaginal birth & Yes/No \\
Caesarian section & Yes/No \\
GDM = gestational diabetes mellitus; BMI = body mass index.
\end{tabular}


median, were used to express results. Univariate analysis was done for determining the factors responsible for GDM. Unadjusted odds ratio (OR) with $95 \%$ confidence interval (CI) was reported and $p<0.05$ considered statistically significant.

\section{Results}

The mean (SD) age of GDM subjects was found to be 29.30 (4.37) years, similar to the matched control group with mean (SD) age of 29.09 (4.01) years. Sociodemographic variables depicted in Table 2 showed $71.37 \%$ of the study subjects were from urban areas, but risk of having GDM was detected to be higher in women from rural areas with lower socioeconomic status. Of the study subjects, $79.91 \%$ had a family income $\leq$ INR20 000 per month. Table 3 shows risk factors based on current pregnancy: women with BMI $>23 \mathrm{~kg} / \mathrm{m}^{2}$ had greater risk of GDM development (OR 2.774; 95\% CI 1.352 - 5.692). The odds of diagnosing GDM is higher when diagnosis of GDM is made at or before 28 weeks of pregnancy (OR 1.976; CI 0.772 - 5.055). Pregnancy-induced hypertension showed an association with GDM (OR 1.31; 95\% CI 0.48 - 3.63), as did polyhydramnios (OR 6.940; 95\% CI 0.793 - 60.778) and oligohydramnios (OR 1.208; 95\% CI 0.387 - 3.774) during initial months of pregnancy, but numbers were possibly too small to show significance.

Table 4 shows the risk factors for GDM based on history of previous pregnancies: gap between pregnancies showed that women having $<2$ years' spacing between pregnancies had a significantly higher risk of developing GDM (OR 2.068; 95\% CI 1.257 - 3.403) women having had abortions in the past were at a higher risk for development of GDM (OR 1.26; 95\% CI $0.69-2.29$ ), as was a history of pre-eclampsia (OR 3.41; 95\% CI 0.49 - 23.36), and preterm labour (OR 1.49; 95\% CI 0.52 - 4.28). On the other hand, women who had completed a full term of pregnancy delivered either vaginally $(p<0.05)$ or by caesarean section $(p<0.05)$ had a diminished risk of GDM.

\section{Discussion}

The present study was conducted to determine the risk factors for GDM in a tertiary healthcare facility in Mangalore. In our study, $71.37 \%$ population were from urban areas and $79.91 \%$ had a monthly income $\leq$ INR 20000 . This may relate to the district hospital setting where costs of healthcare services are minimal. Women from rural areas (OR 1.510; 95\% CI 0.385 - 1.135) and women who had a monthly income $\leq$ INR 20000 per month (OR 1.149; 95\% CI 0.605 - 2.180) had a higher risk of developing GDM, although in the latter category this was not significant. A study conducted in Tamil Nadu detected GDM in $17.8 \%$ of urban women, $13.8 \%$ in semi-urban women and $9.9 \%$ in women from rural areas, showing the prevalence of GDM to be higher in urban areas than rural areas. ${ }^{[10]}$

Pre-pregnancy BMI is one of the important determinants of GDM. $\cdot^{[2,12,14,16]}$ In our study we found a cut-off value of BMI $>23 \mathrm{~kg} / \mathrm{m}^{2}$ (OR $2.774,95 \%$ CI 1.352 - 5.692) to be significantly associated with GDM. A similar hospital-based case-control study conducted in Delhi also found a BMI $>23 \mathrm{~kg} / \mathrm{m}^{2}$ to be significantly associated with GDM. ${ }^{[16]}$ As per the World Health Organization (WHO) recommended Asian classification of BMI, this value

Table 2. Unadjusted OR for each risk factor analysed based on sociodemographic information - the results of univariate analysis

\begin{tabular}{|c|c|c|c|c|}
\hline & Cases $(N=131), n(\%)$ & Control $(N=131), n(\%)$ & OR (CI 95\%) & $p$-value \\
\hline \multicolumn{5}{|c|}{ Residential location } \\
\hline Rural & $43(32.8)$ & $32(24.4)$ & $1.51(0.385-1.135)$ & 0.133 \\
\hline Urban & $88(67.2)$ & $99(75.6)$ & & \\
\hline \multicolumn{5}{|c|}{ Monthly family income (INR)* } \\
\hline$\leq 20000$ & $94(81.0)$ & $93(78.8)$ & $1.149(0.605-2.180)$ & 0.672 \\
\hline$>20000$ & $22(19.0)$ & $25(21.2)$ & & \\
\hline
\end{tabular}

Table 3. Unadjusted OR for each risk factor analysed based on current pregnancy - the results of univariate analysis

\begin{tabular}{|c|c|c|c|c|}
\hline & Cases $(N=131)^{\star}, n(\%)$ & Controls $(N=131)^{\star}, n(\%)$ & OR (CI 95\%) & $p$-value \\
\hline \multicolumn{5}{|c|}{ BMI at GDM diagnosis $\left(\mathrm{kg} / \mathrm{m}^{2}\right)$} \\
\hline$>23$ & $38(55.1)$ & $19(30.6)$ & $2.774(1.352-5.692)$ & 0.005 \\
\hline$\leq 23$ & $31(44.9)$ & $43(69.4)$ & & \\
\hline \multicolumn{5}{|c|}{ Period of gestation at GDM diagnosis (weeks) } \\
\hline$\leq 28$ & $12(12.8)$ & $8(6.9)$ & $1.976(0.772-5.055)$ & 0.150 \\
\hline$>28$ & $82(87.2)$ & $108(93.1)$ & & \\
\hline Yes & $8(12.9)$ & $9(10.1)$ & $1.317(0.478-3.626)$ & 0.594 \\
\hline No & $54(87.1)$ & $80(89.9)$ & & \\
\hline \multicolumn{5}{|c|}{ Polyhydramnios } \\
\hline Yes & $8(12.9)$ & $9(10.1)$ & $6.940(0.793-60.778)$ & 0.044 \\
\hline No & $54(87.1)$ & $80(89.9)$ & & \\
\hline \multicolumn{5}{|c|}{ Oligohydramnios } \\
\hline No & $61(91.0)$ & $86(92.5)$ & & \\
\hline
\end{tabular}


RESEARCH

Table 4. Unadjusted OR for each risk factor analysed based on previous pregnancies - the results of univariate analysis

\begin{tabular}{|c|c|c|c|c|}
\hline & Cases $(N=131)^{\star} n(\%)$ & Control $(N=131)^{*} n(\%)$ & OR (CI 95\%) & $p$-value \\
\hline \multicolumn{5}{|c|}{ Mean gap between pregnancies (years) } \\
\hline$<2$ & $75(59.1)$ & $52(40.9)$ & $2.068(1.257-3.403)$ & 0.004 \\
\hline$\geq 2$ & $53(41.1)$ & $76(58.9)$ & & \\
\hline \multicolumn{5}{|l|}{ Abortions } \\
\hline Yes & $30(23.1)$ & $25(19.2)$ & $1.260(0.693-2.290)$ & 0.448 \\
\hline No & $100(76.9)$ & $105(80.8)$ & & \\
\hline \multicolumn{5}{|c|}{ Pre-eclampsia } \\
\hline Present & $3(21.4)$ & $2(7.4)$ & $3.409(0.498-23.361)$ & 0.193 \\
\hline Absent & $11(78.6)$ & $25(92.6)$ & & \\
\hline \multicolumn{5}{|c|}{ Pre-term labour } \\
\hline Yes & $8(9.27)$ & $7(6.4)$ & $1.490(0.518-4.283)$ & 0.457 \\
\hline No & $79(90.8)$ & $103(93.6)$ & & \\
\hline \multicolumn{5}{|c|}{ Vaginal birth } \\
\hline Yes & $32(24.4)$ & 47 (35.9) & $0.578(0.338-0.986)$ & 0.043 \\
\hline No & $99(75.6)$ & $84(64.1)$ & & \\
\hline \multicolumn{5}{|c|}{ Caesarean section } \\
\hline Yes & $12(9.2)$ & $22(16.8)$ & $0.500(0.236-1.058)$ & 0.066 \\
\hline No & $119(90.8)$ & $109(83.2)$ & & \\
\hline
\end{tabular}

corresponds to overweight $\left(23.0 \mathrm{~kg} / \mathrm{m}^{2}-27.5 \mathrm{~kg} / \mathrm{m}^{2}\right){ }^{[17]}$ This suggests that counselling and weight reduction measures before conception may reduce GDM.

Our study indicated that a mean gap between pregnancies of $<2$ years showed a positive correlation (OR 2.068; 95\% CI 1.257 - 3.403) and statistically significant risk for GDM, projecting that an adequate gap between pregnancies might reduce the risk of GDM unless this is not a causative variable but an association. If a causative effect were true, family planning could be an integral part in reducing GDM. Pregnancy-induced hypertension (OR 1.317; 95\% CI 0.478 - 3.626) and pre-eclampsia during previous pregnancies also had a positive association (OR 3.409; 95\% CI 0.498 - 23.361) in our study, although this was not statistically significant. This would, if significant, be in agreement with the findings of previous studies. ${ }^{[10,12,16]}$

Previous mode of delivery was significantly associated with GDM. A previous normal vaginal delivery (OR 0.578; $95 \%$ CI 0.338 - 0.986) showed a negative association with developing GDM. Caesarean section (OR 0.500; 95\% CI 0.236 - 1.058) showed some protection although it was not statistically significant. Factors such as previous miscarriages (OR 1.260; 95\% CI 0.693 - 2.290) or pre-term labour (OR 1.490; 95\% CI 0.518 - 4.283) were found to be possible risk factors for developing GDM although they were not statistically significant. These findings suggest that pregnancies which were completed until term may have had a protective effect against developing GDM; a larger study is required to evaluate whether this would achieve significance. In a study performed in China it was speculated that there is a possible association between miscarriage and the risk of GDM because of common risk factors or shared pathology. ${ }^{[18]}$ However, mechanisms underlying such an association are unclear and demand further investigation.

In this study, $12.8 \%$ of cases of GDM were identified before 28 weeks of gestation - the time when pregnant women are screened for GDM as per established protocol norms. ${ }^{[2]}$ The remainder, $87.2 \%$ of cases, were diagnosed either after 28 weeks of gestation or late in the third trimester. There is, therefore, a need to diagnose a significant number of impaired glucose tolerance and GDM cases early during pregnancy to enable provision of intervention and treatment.

Regarding previous pregnancy outcomes, 9.27\% of GDM cases had previous pre-term labour (OR 1.490; 95\% CI 0.518 - 4.283) (not significant) and $8.3 \%$ had in the past had either polyhydramnios (OR 6.940; 95\% CI 0.793 - 60.778) (not significant) or oligohydramnios (OR 1.208; 95\% CI 0.387 - 3.774) (not significant). A similar study in South India reported these factors as significant for developing GDM. ${ }^{[10]}$

\section{Study limitations}

Since it is a single-centre study, the findings may only be generalised to the GDM patients of a district maternity hospital.

There may be selection bias in this case-control study by virtue of its being hospital based. Incorporation of face-to-face interviews with patients might have given more complete information. Unknown factors may have a confounding effect on findings and some suggestions have been made regarding factors which did not reach significance. This warrants a cohort study of greater size.

The present study provides information that, in spite of these constraints, may be beneficial in assisting pregnancy care. Adequate pre-pregnancy counselling for mothers-to-be, early recognition and providing quality affordable prenatal healthcare to GDM-affected women, along with incorporation of screening programmes for high-risk pregnant women, are important in healthcare planning as they emphasise early intervention, control and prevention of modifiable risk factors during the pre-conception and antenatal period. Therapeutic intervention with corrective measures such as dietary modification and exercise, emphasising daily physical activities, could lead to a reduced incidence of the feto-maternal morbidity associated with GDM.

\section{Conclusion}

According to the findings of our study, BMI $>23 \mathrm{~kg} / \mathrm{m}^{2}$ and $<2$ years of spacing between pregnancies were significant risk factors. Vaginal 
birth as the previous mode of delivery was found to have significant protective effect against developing GDM. Gestational diabetes is a major health problem in the world that necessitates effective prevention and control strategies. Thus, availability of accurate and reliable information regarding the causes is essential in planning the prevention and treatment of GDM.

\section{Declaration. None.}

Acknowledgements. None.

Author contributions. BU, PR and SKB were involved in developing the idea, design, planning, conduct, data analysis, manuscript writing, assessment and review of final version of manuscript. PHN was involved in in the design, planning, conduct and data analysis. AS and $\mathrm{RN}$ were involved in the design, planning and conduct. PO contributed the idea of the topic, and was involved in planning and design.

\section{Funding. None.}

\section{Conflicts of interest. None.}

1. Metzger BE, Buchanan TA, Coustan DR, et al. Summary and recommendations of the Fifth International Workshop-Conference on Gestational Diabetes Mellitus. Diabetes Care 2007;30(Suppl 2):S251-260

2. American Diabetes Association. Comprehensive medical evaluation and assessment of comorbidities: Standards of medical care in diabetes - 2018. Diabetes Care 2018;41(Suppl 1):S28 37. https://doi.org/10.2337/dc18-S003

3. Morisset AS, St-Yves A, Veillette J, Weisnagel SJ, Tchernof A, Robitaille J. Prevention of gestational diabetes mellitus: A review of studies on weight management. Diabetes Metab Res Rev 2010;26(1):17-25. https://doi.org/10.1002/dmrr.1053

4. Chan JCN, Malik V, Jia W, et al. Diabetes in Asia: Epidemiology, risk factors, and pathophysiology. JAMA 2009;301(20):2129-2140. https://doi.org/10.1001/jama.2009.726.

5. Lee KW, Ching SM, Ramachandran V, et al. Prevalence and risk factors of gestational diabetes mellitus in Asia: A systematic review and meta-analysis. BMC Pregnancy Childbirth 2018;18(1):120. https://doi.org/10.1186/s12884-018-2131-4
6. Tandon N, Anjana RM, Mohan V, et al. The increasing burden of diabetes and variations among the states of India: The Global Burden of Disease Study 1990 - 2016. Lancet Glob Health 2018;6(12):e1352-1362

7. Whiting DR, Guariguata L, Weil C, Shaw J. IDF Diabetes Atlas: Global estimates of the prevalence of diabetes for 2011 and 2030. Diabetes Res Clin Pract 2011;94(3):311-321.

8. Raja MW, Baba TA, Hanga AJ, Bilquees S, Rasheed S, Haq IU, Khan SS, Bashir A. A study to estimate the prevalence of gestational diabetes mellites in an urban block of Kashmir valley (North India). Int J Med Sci Public Health $2014 ; 3(2): 191-195$.

9. Swami SR, Mehetre R, Shivane V, Bandgar TR, Menon PS, Shah NS. Prevalence of carbohydrate intolerance of varying degrees in pregnant females in Western India (Maharashtra): A hospitalintolerance of varying degrees in pregnant females in
based study. I Indian Med Assoc 2008;106(11):712-714.

10. Krishnaveni GV, Hill JC, Veena SR, et al. Gestational diabetes and the incidence of diabetes in the 5 years following the index pregnancy in South Indian women. Diabetes Res Clin Pract 2007;78(3):398-404.

11. Seshiah V, Balaji V, Balaji MS, Paneerselvam A, Kapur A. Pregnancy and diabetes scenario around the world: India. Int J Gynecol Obstet 2009;104(Suppl 1):S35-38. https://doi.org/10.1016/j. ijgo.2008.11.035.

12. Arora GP, Thaman RG, Prasad RB, et al. Prevalence and risk factors of gestational diabetes in Punjab, North India: Results from a population screening program. Eur J Endocrinol 2015;173(2):257-267

13. Bhat M, Ramesha KN, Sarma SP, Menon S, Sowmini CV, Ganesh Kumar S. Determinants of gestational diabetes mellitus: A case control study in a district tertiary care hospital in south India. Int J Diabetes Dev Ctries 2010;30(2):91-96. https://doi.org/10.4103/0973-3930.62599.

14. Gopalakrishnan V, Singh R, Pradeep Y, et al. Evaluation of the prevalence of gestational diabetes mellitus in North Indians using the International Association of Diabetes and Pregnancy Study mellitus in North Indians using the International Association of Diabetes and Pregnancy Study
groups (IADPSG) criteria. J Postgrad Med 2015;61(3):155-158. https://doi.org/10.4103/0022groups (IAD

15. Pramanick S, Panda P. Study of the effects of gestational diabetes on pregnant mothers and their new-born babies in a medical college in West Bengal. Inter J Contemp Med Res 2017;4(6). https:// www.ijcmr.com/uploads/7/7/4/6/77464738/ijcmr_1486_jun_28.pdf (accessed 23 July 2020).

16. Seshiah V, Balaji V, Balaji MS, Sanjeevi CB, Green A. Gestational diabetes mellitus in India. J Assoc Physicians India 2004;52:707-711.

17. Nishida C, Barba C, Cavalli-Sforza T, et al. Appropriate body-mass index for Asian populations and its implications for policy and intervention strategies. Lancet 2004;363(9403):157-163. https://doi. org/10.4103/0022-3859.159306

18. Nanda S, Savvidou M, Syngelaki A, Akolekar R, Nicolaides KH. Prediction of gestational diabetes mellitus by maternal factors and biomarkers at 11 to 13 weeks. Prenat Diagn 2011;31(2):135-141. https://doi.org/10.1002/pd.2636

Accepted 24 July 2020. 